\title{
WKB Wave Functions with the Induced Gravity Theory
}

\author{
Zong-Hong Zhu* and Li Cao ${ }^{\dagger}$ \\ Beijing Astronomical Observatory, Chinese Academy of Sciences, Beijing 100012, China \\ National Astronomical Observatories, Chinese Academy of Sciences, Beijing 100012, China
}

\begin{abstract}
The Wheeler-DeWitt equation for the induced gravity theory is constructed in the minisuperspace approximation, and then solved using the WKB method under three types of boundary condition proposed respectively by Hartle \& Hawking ("no boundary"), Linde and Vilenkin ("tunneling from nothing"). It is found that no matter how the gravitational and cosmological "constants" vary in the classical models, they will acquire constant values when the universe comes from quantum creation, and that, in particular, the resulting tunneling wave function under the Linde or Vilenkin boundary condition reaches its maximum value if the cosmological constant vanishes.
\end{abstract}

*e-mail address: zhuzh@class1.bao.ac.cn

†e-mail address: caoli@nova.bao.ac.cn 


\section{INTRODUCTION}

It is known that the Hartle-Hawking quantum cosmology is based on Einstein's general relativity. Although the latter is extremely successful at describing the observable universe, it doesn't fully incorporate Mach's principle, which demands that spacetime is determined entirely by background matter fields and physical laws. Hence the other type of theories, notably the Brans-Dicke theory [1] and the induced gravity theory [2], in which the gravitational and cosmological "constants" can result from a scalar field. In such a gravity theory, both the the gravitational and cosmological "constants" are dynamical and timedependent quantities. Observationally, there exist a number of experimental constraints on the time variation of the gravitational "constant", $G$ [3], of which the tightest bound, $|\dot{G} / G|=(0.6 \pm 2.0) \times 10^{-12} \mathrm{yr}^{-1}$, was found by Thorsett 团 using Bayesian statistical techniques on the measurements of the masses of young and old neutron stars in pulsar binaries. The upper limits on the time variation of the cosmological "constant", $\Lambda$, can be deduced from number counts of faint galaxies [5], statistical properties of gravitational lensing [6], structure formation [7] and other ways. All results indicate an almost constant $\Lambda$. Moreover, many attempts have been made in order to develop a plausible model in which the cosmological constant $\Lambda$ is set to be precisely zero [8,9]. So a critical problem is how the universe acquires almost constant values for $G$ and $\Lambda$, and especially, a vanishing value for the latter. Because quantum cosmology (for an elegant review, see Ref. [10]) could, with no more than physical laws, provide a scheme which explains the present universe is what it is, we should endeavor to solve the problems mentioned above within the framework of such theory. In this context, We have considered the Brans-Dicke theory in previous papers [11, here we consider the induced gravity theory.

The first consideration of the quantum cosmology based on the induced gravity theory was given by Mo and Fang using the path integral technique [12]. In this letter, we try to tackle the problem using the canonical quantization method, concentrating particularly on the time variation of $G$ and $\Lambda$. The Wheeler-Dewitt equation (WDWE) is constructed 
in the minisuperspace approximation and the wave functions of the universe are obtained using three kinds of boundary condition, that are proposed by Hartle \& Hawking [13], Linde [14 and Vilenkin [15] respectively. We shall show that no matter how $G$ and $\Lambda$ vary in the classical models, they will acquire constant values when the universe comes from quantum creation. Moreover, the amplitude of the resulting wave function under the Vilenkin or Linde boundary condition sharply peaks around the classical trajectory only for a vanishing cosmological constant.

\section{THE WHEELER-DEWITT EQUATION FOR THE INDUCED GRAVITY THEORY}

The action of induced gravity is [2, 12$]$

$$
S=\int d^{4} x \sqrt{-g}\left[\frac{1}{2} \epsilon \varphi^{2} R-\frac{1}{2} g^{\mu \nu} \varphi_{, \mu} \varphi_{, \nu}-V(\varphi)\right]+\ldots
$$

So the scalar field $\varphi$ induces a universe where the gravitational and cosmological "constants" are given simutaneously by

$$
\begin{array}{r}
\left(16 \pi G_{\text {ind }}\right)^{-1}=\frac{1}{2} \epsilon \varphi^{2}, \\
\Lambda_{\text {ind }}=\frac{V(\varphi)}{\epsilon \varphi^{2}} .
\end{array}
$$

We will not assume a specific form for $V(\varphi)$, except that it is of the induced gravity type, for example, $V(\varphi)=\frac{\lambda}{8}\left(\varphi^{2}-v^{2}\right)^{2}$. Note that $\epsilon, \lambda$, and $v$ are all small constants. For quantum cosmology, gravitation is always dominant, and we may neglect terms representing other fields in the action, Eq.1. Under the minisuperspace approximation, the metric of spacetime is given by

$$
d s^{2}=-N(t)^{2} d t^{2}+a(t)^{2} d \Omega_{3}^{2}
$$

where $d \Omega_{3}^{2}$ is the line element of the three dimensional unit sphere, $N(t)$ is the lapse function. The scalar gravitational field $\varphi$ depends on $t$ only. The total action can thus be written as 


$$
S=\int d t 2 \pi^{2}\left[-3 \epsilon \frac{a}{N} \varphi^{2} \dot{a}^{2}-6 \epsilon \frac{a}{N} a \varphi \dot{a} \dot{\varphi}+\frac{1}{2} \frac{a}{N} a^{2} \dot{\varphi}^{2}+3 \epsilon \frac{N}{a} a^{2} \varphi^{2}-\frac{N}{a} a^{4} V(\varphi)\right]
$$

where the dot stands for derivatives with respect to $t$. The momenta conjugate to $a$ and $\varphi$ are defined in usual way and are respectively given by

$$
\begin{aligned}
& \Pi_{a} \equiv \frac{\delta S}{\delta \dot{a}}=2 \pi^{2}\left(-6 \epsilon \frac{a}{N} \varphi^{2} \dot{a}-6 \epsilon \frac{a}{N} a \varphi \dot{\varphi}\right) \\
& \Pi_{\varphi} \equiv \frac{\delta S}{\delta \dot{\varphi}}=2 \pi^{2}\left(-6 \epsilon \frac{a}{N} a \varphi \dot{a}+\frac{a}{N} a^{2} \dot{\varphi}\right)
\end{aligned}
$$

Then we have the following relations,

$$
\begin{aligned}
& a \dot{\varphi}=\frac{N}{2 \pi^{2} a} \frac{\varphi \Pi_{\varphi}-a \Pi_{a}}{(6 \epsilon+1) a \varphi}, \\
& \dot{a} \varphi=-\frac{N}{2 \pi^{2} a} \frac{6 \epsilon \varphi \Pi_{\varphi}+a \Pi_{a}}{6 \epsilon(6 \epsilon+1) a \varphi} .
\end{aligned}
$$

Taking the variation of the action, Eq.⿴囗十, with respect to the lapse function $N$ and combining with Eq.6, we obtain the Hamiltonian constraint for the induced gravity theory,

$H=-\left(12 \epsilon(6 \epsilon+1) a \varphi^{2}\right)^{-1}\left(\Pi_{a}^{2}-6 \epsilon \frac{\varphi^{2}}{a^{2}} \Pi_{\varphi}^{2}+12 \epsilon \frac{\varphi}{a} \Pi_{a} \Pi_{\varphi}\right)-\left(2 \pi^{2}\right)^{2} a\left[3 \epsilon \varphi^{2}-a^{2} V(\varphi)\right]=0$.

By introducing the canonical quantization into the above Hamiltonian constraint, $\Pi_{a} \rightarrow$ $\frac{1}{i} \frac{\partial}{\partial a}, \Pi_{\varphi} \rightarrow \frac{1}{i} \frac{\partial}{\partial \varphi}$, we obtain the WDWE for the induced gravity theory,

$$
\left\{\frac{\partial^{2}}{\partial a^{2}}-6 \epsilon \frac{\varphi^{2}}{a^{2}} \frac{\partial^{2}}{\partial \varphi^{2}}+12 \epsilon \frac{\varphi}{a} \frac{\partial}{\partial a} \frac{\partial}{\partial \varphi}+48 \pi^{4} \epsilon(6 \epsilon+1) a^{2} \varphi^{2}\left[a^{2} V(\varphi)-3 \epsilon \varphi^{2}\right]\right\} \Psi(a, \varphi)=0 .
$$

In constructing the WDWE (Eq.8), we have ignored the operator order problem which is not important in the following discussion.

\section{WKB WAVE FUNCTIONS OF THE UNIVERSE}

In order to make predictions, we should solve the WDWE Eq.8, which proves to be a very difficult task. A regulous solution seems difficult, so we look for some simple solutions that do not depend sensitively on $\varphi$. We neglect the second and third terms in Eq.8 to get 
cosmic wave functions. The problem is then simplified to a standard one-dimensional WKB problem for the scale factor $a$ with a potential

$$
U(a)=48 \pi^{4} \epsilon(6 \epsilon+1) a^{2} \varphi^{2}\left[3 \epsilon \varphi^{2}-a^{2} V(\varphi)\right],
$$

For the classicall allowed (oscillatory) region $a \geq a_{H} \equiv\left[3 \epsilon \varphi^{2} / V(\varphi)\right]^{1 / 2}$, where the scale factor is large, there are WKB solutions of the form

$$
\begin{aligned}
\Psi_{ \pm}(a, \varphi) & =[p(a)]^{-1 / 2} \exp \left[ \pm i \int_{a_{H}}^{a} p\left(a^{\prime}\right) d a^{\prime} \mp i \pi / 4\right] \\
& =\left[4 \pi^{2} \sqrt{3 \epsilon(6 \epsilon+1)} a \varphi \sqrt{a^{2} V(\varphi)-3 \epsilon \varphi^{2}}\right]^{-1 / 2} \exp \left[ \pm i \frac{4 \pi^{2} \sqrt{3 \epsilon(6 \epsilon+1)} \varphi}{3 V(\varphi)}\left[a^{2} V(\varphi)-3 \epsilon \varphi^{2}\right]^{3 / 2} \mp i \pi / 4\right], \quad a \geq a_{H},
\end{aligned}
$$

where $p(a)=[-U(a)]^{1 / 2}$. For the classically forbiden (exponential) region $a<a_{H}$, where the scale factor is small, there are WKB solutions of the form

$$
\begin{aligned}
\tilde{\Psi}_{ \pm}(a, \varphi) & =|p(a)|^{-1 / 2} \exp \left[ \pm \int_{a}^{a} H\left|p\left(a^{\prime}\right)\right| d a^{\prime}\right] \\
& =\left[4 \pi^{2} \sqrt{3 \epsilon(6 \epsilon+1)} a \varphi \sqrt{\left(3 \epsilon \varphi^{2}-a^{2} V(\varphi)\right)}\right]^{-1 / 2} \exp \left[ \pm \frac{4 \pi^{2} \sqrt{3 \epsilon(6 \epsilon+1)} \varphi}{3 V(\varphi)}\left[3 \epsilon \varphi^{2}-a^{2} V(\varphi)\right]^{3 / 2}\right], \quad a \leq a_{H} .
\end{aligned}
$$

We can impose the boundary condition in either the classically allowed region or the classically forbidden region, and then match the solutions in the two regions by the WKB standard matching procedure to specify the WKB wave function.

There are several comprehensive and well studied boundary condition proposals in the literature (for a recent review, see Ref. [16]). Now we seek for the specified WKB wave function with different boundary conditions following Vilenkin [16]. Hartle \& Hawking [13] proposed that the specified wave function should be given by the Euclidean path integral, $\Psi_{H H}=\int e^{-S_{E}}$, which is taken over compact Euclidean geometries and matter fields with a specified field configuration at the boundary. Note that $S_{E}$ is the Euclidean action. The Hartle-Hawking wave function is specified by requiring that it is given by $\exp \left(-S_{E}\right)$ in the Euclidean regime. This gives 13

$$
\begin{aligned}
& \Psi_{H H}\left(a<a_{H}, \varphi\right)=\tilde{\Psi}_{-}(a, \varphi), \\
& \Psi_{H H}\left(a>a_{H}, \varphi\right)=\Psi_{+}(a, \varphi)-\Psi_{-}(a, \varphi) .
\end{aligned}
$$

However, Linde [14 argued that the wave function should be given by, $\Psi_{L}=\int e^{+S_{E}}$, which requires a reverse sign of the exponential in the Euclidean regime. Together with the continuation to the classically allowed range of $a$, one can get the Linde wave function as 


$$
\begin{aligned}
& \Psi_{L}\left(a<a_{H}, \varphi\right)=\tilde{\Psi}_{+}(a, \varphi), \\
& \Psi_{L}\left(a>a_{H}, \varphi\right)=\frac{1}{2}\left[\Psi_{+}(a, \varphi)+\Psi_{-}(a, \varphi)\right] .
\end{aligned}
$$

In addition, Vilenkin suggested that the wave function of the universe should be specified either by the tunneling boundary condition [15] or by a Lorentzian path integral [17], $\Psi_{V}=$ $\int e^{i S}$. Let us give a more precise statement of the Vilenkin's tunneling boundary condition as follows [10, [18]: $\Psi_{V}$ is the solution to the WDWE that is everywhere bounded and only consists of outgoing modes at singular boundaries of superspace. The superspace for our model is two-dimensional space with coordinates $(a, \varphi)$, where, $0<a<\infty,-\infty<\varphi<\infty$. The unique non-singular part of the boundary is $a=0$ with $\varphi$ being finite. The rest are singular and consist of configuration with one or both $a$ and $\varphi$ being infinite. Note that as $a$ approaches zero, the coefficients of the second and third terms in Eq.8 blow up. As the boundary condition requires, we are to get a regular solution, it seems reasonable to neglect the second and third terms in Eq.8 to get the wave function of the universe. It is easy to check that $\Psi_{-}(a, \varphi)$ and $\Psi_{+}(a, \varphi)$ describe an expanding and a contracting universe, respectively. Vilenkin's boundary condition requires that only the expanding component $\Psi_{-}(a, \varphi)$ should be present at large $a$. The wave function within the quantum barrier can be found from the WKB connection formula. Finally, we get the Vilenkin wave function,

$$
\begin{aligned}
& \Psi_{V}\left(a<a_{H}, \varphi\right)=\tilde{\Psi}_{+}(a, \varphi)-\frac{i}{2} \tilde{\Psi}_{-}(a, \varphi), \\
& \Psi_{V}\left(a>a_{H}, \varphi\right)=\Psi_{-}(a, \varphi) .
\end{aligned}
$$

This completes the calculation of the wave function of the universe under different types of boundary condition.

\section{RESULT AND DISCUSSION}

Unfortunately, there has been no consensus so far on the interpretation of the wave function of the universe among the quantum cosmology community, except the statement that when $\Psi \sim \exp [i S / \hbar]$, classical behavior should be recovered [19]. In order to understand the 
physical meaning of the resulting wave functions, we employ the so-called "peak interpretation", in which a prediction is said to be made when the wave function is sharply peaked in a certain region and almost zero elsewhere [20,21]. It is worthwhile to point out that, the causal or the the Bohm-de Broglie interpretation will lead to the same result [21].

For the classical region, $a \geq a_{H}$, where spacetime has the classical meaning and the classical solutions are valid, all three kinds of wave function are essentially oscillating, so the probability distribution does not depend sensitively on $a$ or $\varphi$. This independence implies that there is approximatively equal probability for each point on the classical trajectories. Therefore, the properties of the wave function of the universe in the quantum era is crucial for the subsequent evolution of the universe. Within the quantum barrier, both Linde's and Vilenkin's wave functions are dominated by the decaying exponential $\tilde{\Psi}_{+}(a, \varphi)$, which piles up at $V(\varphi)=0$, i.e., $\varphi^{2}=v^{2}$, in the case of $V(\varphi)=\frac{\lambda}{8}\left(\varphi^{2}-v^{2}\right)^{2}$. Since $\epsilon$ is small, the distribution will be concentrated in the narrow region around $\varphi^{2}=v^{2}$. Therefore the gravitational and cosmological "constants", $G_{\text {ind }}, \Lambda_{\text {ind }}$, acquire constant values, and especially, the latter is equal to zero. It implies that no matter how the cosmological constant can vary in the classical models, it will posses zero value when the universe comes from quantum creation. Nevertheless, Hartle-Hawking wave function within the barrier contain only the growing exponential $\tilde{\Psi}_{-}(a, \varphi)$, which piles up at $V(\varphi)=V_{\max }$, where $V_{\max }$ is the maximum value of $V(\varphi)$. Hence the universe would prefer a large cosmological constant.

In summary, we have investigated a quantum cosmological model with the induced gravity theory. After the WDWE was constructed in the minisuperspace approximation, we have solved it using three kinds of boundary condition. We have shown that the amplitude of the resulting tunneling wave function sharply peaks around the classical trajectory only for a vanishing cosmological constant.

\section{ACKNOWLEDGMENTS}

We thank the anonymous refree for valuable comments, Prof. L. M. Krauss for help- 
ful suggestions and Prof. T. Kiang of Dunsink Observatory, Ireland, for polishing up the English. This work was supported by the National Natural Science Foundation of China, under Grant No. 19903002. 


\section{REFERENCES}

[1] C. Brans and R. Dicke, Phys. Rev. 124, 925(1961).

[2] A. Zee, Phys.Rev.Lett. 42, 417(1979).

[3] L. M. Krauss and M. White, 1992, Astrophys. J., 397, 357(1992); G. T. Gillies, Rep. Prog. Phys. 60, 151(1997).

[4] S. E. Thorsett, Phys.Rev.Lett. 77 1432(1996).

[5] Y. Yoshii and K, Sato, Astrophys. J., 387, L7(1992).

[6] L. F Bloomfield Torres and I. Waga, MNRAS, 279, 712(1996).

[7] P. T. P. Viana and A. R. Liddle, Phys. Rev. D57, 674(1998).

[8] S. Weinberg, Rev. Mod. Phys. 61, 1(1989).

[9] S. M. Carroll, W. H. Press and E. L. Turner, Ann. Rev. Astron. Astrophys. 30, 499(1992).

[10] J. J. Halliwell, "An Introduction on Quantum Cosmology", in: Proceedings of Jerusalem Winter School on Quantum Cosmology and Baby Univers e, ed. by S. Coleman, J. B. Hartle, T. Piran and S. Weinberg (Singapore: World Scientific, 1991).

[11] Z. H. Zhu, C. G. Huang and L. Liu, Chin. Phys. Lett 7, 477(1990); Z. H. Zhu, ibid. 9, 273(1992); Z. H. Zhu, Y. Z. Zhang and X. P. Wu, Mod.Phys.Lett. A13, 1333(1998).

[12] H. J. Mo and L. Z. Fang, Phys. Lett. B201, 321(1988).

[13] J. B. Hartle and S. W. Hawking, Phys. Rev. D28, 2960 (1983).

[14] A. D. Linde, Lett. Nuovo Cimento 39, 401(1984).

[15] A. Vilenkin, Phys. Rev. D33, 3560(1986); D37 888(1988).

[16] A. Vilenkin, Phys. Rev. D58, 067301(1998). 
[17] A. Vilenkin, Phys. Rev. D30, 509(1984).

[18] N. Pinto-Neto, "Quantum Cosmology", in: Cosmology and Gravitation II, ed. by M. Novello (Editions Frontieres, Gyf-sur-Yvette, 1996).

[19] E. W. Kolb and M. S. Turner, The Early Universe, p457 (Addison-Wesley Publishing Company, 1990).

[20] J. J. Halliwell, Phys. Rev. D36, 3626(1987).

[21] J. Acácio de Barros and N. Pinto-Neto, Nucl. Phys. B (Proc. Suppl.) 57, 247(1997). 